\title{
Diminishing Effects After Recurrent Use of Self-Guided Internet-Based Interventions in Depression: Randomized Controlled Trial
}

Lara Bücker ${ }^{1}$, MSc; Patricia Schnakenberg ${ }^{1}$, MSc; Eirini Karyotaki ${ }^{2}$, PhD; Steffen Moritz ${ }^{1}$, Prof Dr; Stefan Westermann ${ }^{1,3}, \mathrm{PhD}$

\footnotetext{
${ }^{1}$ Department of Psychiatry and Psychotherapy, University Medical Center Hamburg-Eppendorf, Hamburg, Germany

${ }^{2}$ Department of Clinical Psychology, VU Amsterdam, Amsterdam, Netherlands

${ }^{3}$ Idiographic Dynamics Lab, Department of Psychology, University of California, Berkeley, CA, United States
}

\section{Corresponding Author:}

Lara Bücker, MSc

Department of Psychiatry and Psychotherapy

University Medical Center Hamburg-Eppendorf

Martinistr 52

Hamburg, 20246

Germany

Phone: 4940741055868

Email:1.buecker@uke.de

\section{Abstract}

Background: Self-guided internet-based interventions have several advantages over guided interventions and are generally effective in treating psychiatric symptoms.

Objective: We aimed to investigate whether the use of a new self-guided internet-based intervention (MOOD) would lead to a significant reduction in depressive symptoms compared with a care-as-usual (CAU) control group in a sample of individuals with depressive symptoms, most of whom had already used a different self-guided internet-based intervention in a previous trial.

Methods: A total of 125 individuals were randomized to the intervention condition (MOOD) and received access to the intervention for a period of six weeks or a CAU group. After six weeks, all participants were invited to take part in the post assessment. The Beck Depression Inventory-II served as the primary outcome.

Results: Both intention-to-treat as well as per-protocol analyses indicated that the depressive symptomatology decreased in both conditions but showed no advantage for those who had used MOOD. Subsequent moderation analyses suggested that those individuals who had less experience with psychotherapy benefitted to a greater extent compared with those with more experience.

Conclusions: Self-guided internet-based interventions are deemed a suitable first-step approach to the treatment of depression. However, our results indicate that they are more efficacious in those with less psychotherapy experience.

Trial Registration: ClinicalTrials.gov NCT03795480; http://clinicaltrials.gov/ct2/show/NCT03795480

(J Med Internet Res 2019;21(10):e14240) doi: 10.2196/14240

\section{KEYWORDS}

eHealth; self-management; depressive symptoms; randomized controlled trial

\section{Introduction}

\section{Background}

Major depression (MD) is one of the most common mental disorders, with more than 300 million people affected worldwide [1]. MD has an aggregated lifetime prevalence of $10.8 \%$ and a 1-year prevalence of $7.2 \%$ [2], and it represents an enormous personal and economic burden $[3,4]$.

There is evidence for the effectiveness of psychotherapy and pharmacotherapy in the treatment of depression [5-7]. Although classic face-to-face psychotherapy is effective, it is not possible to treat all affected individuals by this method. According to the World Health Organization (WHO), the treatment gap for 
depression is $56.3 \%$ [8]. This means that a large number of individuals suffering from depressive symptoms remain untreated. This treatment gap can be attributed to several different causes. One of the reasons being that there is an insufficient number of therapists, resulting in long waiting lists. In rural areas, psychologists and psychiatrists are especially underrepresented [9]. Furthermore, general practitioners often do not recognize $\mathrm{MD}$, and misdiagnoses are frequent [10]. Besides these barriers on the supply side, there are also barriers on the demand side. These include patient fear about stigmatization, (expected) discomfort discussing one's own mental health problems, the wish to overcome problems by oneself, a lack of awareness of the need for help, and the anticipated high cost of treatment [11,12]. In addition, it has been shown that greater levels of depression are associated with increased perceived barriers to seeking help, as depression is linked to deficient motivation and reduced activity, as well as to negative cognitive biases (eg, a negative view of the world or future) [13].

\section{The Potential of Internet-Based Interventions}

Internet-based interventions can help overcome the supply and demand barriers of conventional face-to-face treatment. Internet-based treatments could reach people who fear stigmatization, are widely accessible (especially for those living in rural areas or those with physical barriers), and provide a high level of privacy $[14,15]$. Furthermore, the individuals themselves can decide when and where they want to use the intervention. In addition, these interventions are usually available at low cost and, thereby, are affordable for individuals with limited financial resources [16]. In recent years, numerous internet-based interventions have been developed and investigated for their feasibility, acceptance, effectiveness, and side effects, most being used in the treatment of depression and anxiety disorders [17]. To date, a wide range of studies has provided evidence of the effectiveness of internet-based interventions. Studies have found effect sizes ranging from small to large [18-25].

Internet-based interventions can be categorized as either unguided or guided. Guided internet-based interventions are supported by a therapist or a trained person (eg, via frequent email correspondence or telephone support). In self-guided internet-based interventions, the patient does not receive any additional human support. Meta-analyses have found similar effect sizes for guided internet-based treatments when compared with classical face-to-face treatment $[18,26]$. According to van Ballegooijen et al [27], the average percentage of completed sessions is similar for guided internet-based interventions when compared with face-to-face therapy, although more individuals complete all sessions of the face-to-face therapy. However, individuals who dropped out from face-to-face therapy only completed $24.5 \%$ of the intervention, whereas noncompleters of guided internet-based interventions completed on average 42.1\%. Many other studies have shown that internet-based interventions often have high dropout rates [28]. A meta-analysis that examined predictors of treatment adherence in self-guided internet-based interventions for depression identified several demographic and psychopathological factors (male gender, low educational background, and comorbid anxiety symptoms) that predict dropout [29].

Another meta-analysis by Richards and Richardson [23] included 19 randomized controlled trials (RCTs) of 10 guided and 9 self-guided interventions for depression. The effect size found for the guided internet-based interventions was 0.78 , and for the self-guided interventions the effect size was 0.36 (Cohen d). A study that directly compared guided and self-guided interventions for depression within an experimental comparison of the same program with or without therapeutic support and a control group found an effect size of 0.66 for the self-guided version and 1.14 for the guided version (outcome: Beck Depression Inventory-II or BDI-II), which showed that although the effects were smaller for self-guided than for guided, interventions without support were still effective [30]. A meta-analysis by Baumeister et al [31] also supports the superiority of guided over unguided interventions in terms of effectiveness; however, the extent of this superiority seems to be significantly smaller than that in most previous studies (especially in depression studies).

The advantage of self-guided internet-based interventions over guided interventions is that they provide increased access to treatment for those who need it, even for individuals who do not meet the full criteria of a disorder, and, at the same time, are affordable and conserve resources [21]. A recent meta-analysis used individualized participant data to estimate aggregated effect sizes in 13 RCTs on self-guided internet-based interventions for depression [29]. This type of meta-analysis is better able to identify the true effects while taking into account the variability of the studies (eg, degree of support, adherence to treatment, and setting). The meta-analysis found an effect size of 0.27 (Hedge $g$ ). Contrary to previous results showing that higher baseline symptoms predict a greater reduction of symptoms after the intervention period [32], baseline depressive symptoms did not moderate treatment outcome. Another important finding was that better adherence was associated with better treatment outcome.

\section{Objective}

Self-guided internet-based interventions have several advantages over guided interventions and generally are effective in treating psychiatric symptoms. However, the question of which individuals benefit the most has not been investigated well enough. The aim of this study (NCT03795480) was to investigate the acceptance and effectiveness of a new self-guided internet-based intervention for depressive symptoms in a sample that had already received a similar intervention in the context of an earlier study [33]. In other words, we aimed to investigate the possible benefit of recurrent use of self-guided internet-based interventions. The intervention, called MOOD, was developed to provide individuals experiencing subjective depressive symptoms with low-threshold, self-directed, anonymous, and free access to an unguided internet-based intervention. Most of our participants had previously used a Web-based self-help program for depression in the framework of another trial [33]. The study thus enabled us to investigate whether people who had already received a similar therapy could still benefit from MOOD. We expected that participants who received access to 
MOOD would show a significant reduction of depressive symptoms (primary outcome: BDI-II) compared with the care-as-usual (CAU) control group. In addition, participants in the intervention group were expected to report a significant increase in self-esteem and quality of life after the intervention period, compared with the control group. Furthermore, willingness to change (as assessed with the University of Rhode Island Change Assessment (URICA) scale [34]) was expected to moderate treatment outcome. Another aim of the study was to examine possible moderators of treatment outcome.

\section{Methods}

\section{Study Design}

The study was a randomized controlled superiority trial with 2 conditions and parallel assignment (1:1). During the intervention period of 6 weeks, the intervention group received access to the internet-based self-help intervention MOOD, whereas the CAU group received access after completion of the post assessment. There were 2 assessment times, baseline and posttreatment (with an intervention period of 6 weeks). The study was approved by the local psychological ethics committee of the Center for Psychosocial Medicine of the University Medical Clinic Hamburg-Eppendorf, Germany (approval number: LEPEK-003) All participants gave Web-based informed consent before participating in the study. The study was conducted in accordance with the Declaration of Helsinki. The 2 assessments did not ask for any personal information except for an anonymous email address (instructions on how to create such an address were given); no names, telephone numbers, or addresses were asked for. Email addresses were kept in a handwritten list and were assigned to participant codes. The email addresses were stored in a safe. All other obtained data were anonymized and stored electronically on password-protected computers. If a participant requests the deletion of his or her data after completion of the study, this could be done if they provide the code word. The program MOOD ensures network security via secure sockets layer encryption. Messages that were sent within the internal message system of MOOD met the required data safety standard.

\section{Procedure}

The study was conducted at the University Medical Center Hamburg-Eppendorf (Germany). At the 2 assessment times, baseline and posttreatment, data were obtained via an internet survey (Enterprise Feedback Suite survey from QuestBack Unipark). The baseline assessment obtained sociodemographic and psychopathological data (see subsection Instruments). After 6 weeks, all participants were invited to take part in the post assessment in which the same psychopathological data as before were assessed. Individuals in the treatment group were asked to provide a subjective evaluation of the internet-based self-help intervention. At the beginning of the post assessment, participants were asked to enter the same email address and individual code (numbers and letters) they had generated during the baseline assessment to ensure correct matching of pre and post data. Participants who completed the post assessment were rewarded with access to 2 mindfulness-based self-help manuals. These manuals contain a series of established relaxation and mindfulness exercises (eg, to increase acceptance and self-esteem) and were sent to the participants as PDF files.

\section{Sample size}

The power analysis for calculating the sample size for an analysis of covariances (ANCOVA) was conducted using $\mathrm{G}^{*}$ Power [35] and revealed a sample size of 128 to detect a medium effect of 0.25 , with alpha $=.05$ and a power of .80 , which was expected based on a meta-analysis of the effectiveness of internet-based cognitive behavioral therapy (CBT) for depression [23].

\section{Recruitment}

The sample was recruited via an internal database of individuals with depression who had previously participated in studies conducted by the principle investigator's unit and had given consent that they could be contacted for further studies. Most of the participants had previously participated in the Effectiveness of Internet-Based Depression Treatment study (EVIDENT) investigating the effectiveness of an internet-based self-help program for mild to moderate depressive symptoms [33]. Study invitations were sent via email providing information on the purpose and procedure of the study. In addition, invitations to the study were posted on online forums on depression and depression information websites. If participants were interested in taking part in the study, they were directed to a Web page from which the baseline assessment could be started. No financial compensation was offered.

\section{Eligibility Criteria}

Participants could be included if they fulfilled the following inclusion criteria: subjective psychological distress with desire for treatment for depressive symptoms (there were no cutoff criteria for depressive symptoms at baseline), aged between 18 and 65 years, internet access, and sufficient command of the German language. Individuals with acute suicidality (assessed at baseline using item 9 on suicidal thoughts of the BDI-II, cutoff $\geq 2$ ) and/or a self-reported lifetime diagnosis of schizophrenia or bipolar disorder were excluded from the study. Participants who were excluded because of acute suicidality were contacted and provided with help offers and telephone numbers that could be contacted in case of acute crisis. Participants with other psychiatric diagnoses were not excluded from the study. All participants were allowed to continue previously started treatments (psychotherapy or pharmacotherapy; access to treatment) and also changes in medication or psychotherapy were allowed during the participation.

\section{Randomization}

Participants were randomly allocated into 1 of 2 conditions according to a randomization plan that was set up by the second author using the software Research Randomizer [36]. Block randomization was used to ensure balance between groups. As the study was conducted on the Web and the participants could actively enroll via Web-based registration, the allocation procedure differed from that in classical clinical trials, where allocation is performed by team members. On the basis of the date and time of completion of the baseline assessment, the participants were allocated to conditions following the 
randomization plan. The allocation rule was 1:1. Participants who were allocated into the intervention group received an email containing information on the program and a link to the login Web page of MOOD as well as individual login data in the form of a code and a password. Participants in the CAU group received an email with the information that they would receive access to MOOD after completion of the post assessment.

\section{Intervention}

During the 6-week intervention period, the intervention group had access to MOOD, an internet-based self-help program targeting depressive symptoms. The program was developed by members of the neuropsychology working group of the University Medical Center Hamburg-Eppendorf and comprised 9 modules (see Table 1). The content of each module is based on CBT techniques and elements of the third wave of CBT.
There is evidence that cognitive restructuring (modules ABC-protocol [A: activating event, B: belief, C: consequence] and modifying thoughts) and behavioral activation (module positive activities) are effective techniques in the treatment of depression [37,38]. In addition, the concept of mindfulness, which has received increasing attention in recent years, is addressed and practiced in a module labeled mindfulness. It has been shown that mindfulness has a beneficial effect on the outcome of psychotherapy [39-41]. Strengthening interpersonal skills and competences is strongly recommended within the treatment of depression [37,42] and, therefore, addressed in 1 module (social competence). It is also evident that depression is associated with sleep disturbances, which should, therefore, be targeted in treatment (module sleep) $[43,44]$. 
Table 1. Overview of MOOD modules.

\begin{tabular}{lll}
\hline Title & Description & Specific skills and exercises \\
\hline Introduction & $\begin{array}{l}\text { Presents the outline and goal of the program; discusses } \\
\text { the interactions between thoughts, emotions, and behav- } \\
\text { ior }\end{array}$ & $\begin{array}{l}\text { Introduction into the principles of the program and strengthen- } \\
\text { ing of the treatment motivation; creation of list of values and } \\
\text { needs according to which the user wants to live; emphasis is } \\
\text { placed on the importance of interaction of thoughts, feelings, } \\
\text { and behaviors }\end{array}$
\end{tabular}

\begin{abstract}
$\mathrm{ABC}$ protocol $^{\mathrm{a}}$
Highlights the importance of one's beliefs in dealing with a specific situation; questions automatic beliefs about a situation and helps in developing new, more helpful beliefs
\end{abstract}

Positive activities

Shows how to integrate positive activities into one's daily routine and achieve one's goals

Self-esteem

Makes the user aware of his or her own strengths and teaches strategies on how to improve his or her selfperception

Presents ways to improve social competences to connect with other individuals and reach goals in social relations

Social competence

Mindfulness

Presents various mindfulness-based relaxation and attention exercises to increase mindfulness in daily life

Uncovers depressive dysfunctional thoughts and explains methods to turn these into more realistic thoughts

Modifying thoughts

Sleep

Shows the importance of sleep quality and gives advice on how to improve sleep hygiene

Relapse prevention

Encourages paying attention to warning signals that might trigger depressive episodes and provides helpful coping strategies
Introduction of the ABC protocol according to Ellis [45]; dysfunctional thought patterns (eg, all-or-nothing thinking and catastrophizing) are explained and converted to more helpful thoughts.

Presentation of lists of possible positive activities and exercises aimed at integrating positive activities into everyday life on a regular basis and planning them in a meaningful and realistic way; setting short- and long-term goals

The user is asked to identify personal sources of self-esteem and search for forgotten strength. Obstacles are addressed that could stand in the way of an increase of self-esteem (eg, unfair comparisons). Presentation of a list of concrete actions to increase self-esteem in everyday life (eg, joy diary)

Definition of social competence and presentation of characteristics of aggressive, unsafe, safe, and friendly behavior [46]

Identification of signs of mindlessness; distinction between evaluations or judgements and observations to learn an inner attitude of conscious perception, neutrality, and acceptance; suggestions on how the user can distance himself from stressful feelings and obstructive thoughts; presentation of several classical mindfulness exercise (eg, body scan) - those are guided in audio files

Using ABC protocols to modify depressive dysfunctional thoughts; presentation of different possibilities to positively influence thoughts, such as making concrete statements, avoiding generalizations, changing perspectives, obtaining other opinions, and questioning situations

Psychoeducation on the development and maintenance of sleep disorders; identification of characteristics of healthy sleep; creation of a personal list of tips for improving sleep behavior

Identification of possible triggers of relapse; presentation of suggestions on how these triggers can be avoided-the user is recommended to balance negative stress with positive activities and identify physical, emotional, cognitive, and behavioral warning signals for stress in oneself (according to a checklist from Kaluza [47]); development of an individual emergency plan

\footnotetext{
${ }^{\mathrm{a}} \mathrm{A}$ : activating event, $\mathrm{B}$ : belief, $\mathrm{C}$ : consequence.
}

All modules include interactive exercises, worksheets, pictures, graphics, videos, and audios that aim to incorporate the participant's experiences and individual problems to increase the identification of the participant with the material and illustrate the content in an appealing way. The participants were free to choose the order of the modules and could work through the modules at their own speed. We have decided on a free choice of modules to give the participants experiences of autonomy and thus to minimize feelings of heteronomy (eg, being patronized; see [48] for a discussion of the role of motives in internet-based interventions). We recommended that they worked through 1 or 2 modules per week. The approximate time to finish a module ranged between 30 and $60 \mathrm{~min}$. There was no direct guidance. However, the participants had the opportunity to contact a moderator in case of technical questions or problems via messaging within the program. This feature was optional, and the moderator did not actively contact the users on his or her own initiative. However, several reminders were sent via email to those participants who did not log into the program during the study. The participants were encouraged to have a look at the program and work with it. A short overview with summaries of the content of the modules was attached. The emails only served as a reminder to login to the program at least once; no therapeutic support was offered. Of the 55 participants $(55 / 62,89 \%)$ of the MOOD group who logged in to the program, $5(5 / 55,9 \%)$ used this feature. A total of 3 
participants contacted the moderator several times -2 had 2 contacts $(2 / 55,4 \%), 1$ had 3 contacts $(1 / 55,2 \%)$. Within the intervention period, none of the participants in the CAU group contacted the study staff.

\section{Instruments}

Psychopathological self-rating questionnaires were assessed at the baseline and post assessments. The BDI-II [49] served as the primary outcome. Secondary outcomes included changes in self-esteem and quality of life as well as the participants' subjective evaluation of MOOD.

\section{Beck Depression Inventory-II}

The BDI-II [49] was used to assess depressive symptom severity over the previous 2 weeks. The self-rating questionnaire comprises 21 items; for each item, the participant is asked to evaluate the severity of the symptom on a rating scale from 0 to 3 , with higher scores indicating more severe depressive symptoms. An overall score of 0 to 13 indicates minimal depression, 14 to 19 indicates mild depression, 20 to 28 indicates moderate depression, and 29 to 63 suggests severe depression. The internal consistency of the BDI-II ranges from 0.79 to 0.90 [50].

\section{Patient Health Questionnaire-9-Depression Module}

Change in depressive symptoms was also assessed with the Patient Health Questionnaire-9 (PHQ-9) [51]. The PHQ-9 is a self-rating questionnaire that comprises 9 items on depression, which can be answered on a 4-point rating scale ranging from $0=$ not at all to $3=$ nearly every day. Sum scores can range from 0 to 27 with the following classifications: none or minimal (0-4), mild (5-9), moderate (10-14), and severe (15-27) depressive symptoms. Results of the questionnaire can assist in determining a diagnosis of MD according to Diagnostic and Statistical Manual of Mental Disorders, 4th Edition, criteria. Its internal consistency ranges from 0.86 to 0.89 [50].

\section{Rosenberg Self-Esteem Scale}

The Rosenberg Self-Esteem (RSE) scale [52] was used to assess self-esteem. The scale comprises 10 statements regarding self-esteem. Participants are instructed to rate how much they agree with the statements on a 4-point Likert scale from strongly agree to strongly disagree. Its internal consistency ranges from 0.77 to 0.88 . In its original form, higher scores reflect less self-esteem; however, in our study we used a reversed rating scale such that higher scores reflect more self-esteem.

\section{World Health Organization Quality of Life-Abbreviated Version}

The WHO Quality of Life-abbreviated version (WHOQOL-BREF) assesses quality of life [53]. It is a short version of the WHOQOL-100, with 26 items. The questionnaire contains 4 different types of 5-point rating scales that ask the participant how much, how complete, how often, how good, or how satisfied he or she felt over the previous 2 weeks. The questionnaire has 4 subscales: physical health, psychological, social relations, and environment. The WHOQOL-BREF has an internal consistency of 0.70 [54].

\section{University of Rhode Island Change Assessment}

The URICA scale is a measure of willingness to change [34] and was used in the baseline survey. It comprises 32 items representing 4 phases of change: precontemplation, contemplation, action, and maintenance. In this study, a total of 9 items were used, selected from the subscales of precontemplation, contemplation, and action. The internal consistency is 0.83 , and the reliability of the test-retest lies between 0.63 and 0.75 . Answers could be given on a 5-point Likert scale ranging from 1 (strong disagreement) to 5 (strong agreement). In addition, a single item on expectation regarding the treatment outcome was added: "At present, how successful do you think the MOOD self-help program will be?" Here, answers could be given on a 9-point Likert scale ranging from 1 (not successful at all) to 9 (very successful).

\section{Subjective Appraisal}

The subjective appraisal of the intervention was assessed with questions we generated, as well as with adaptions of the Fragebogen zur Patientenzufriedenheit ZUF-8 (questionnaire to measure patient satisfaction [55]). The internal consistency of the ZUF-8 ranges between 0.87 and 0.93 . Items could be answered on a 4-point Likert scale ranging from totally disagree to totally agree. Open questions gave participants the opportunity to provide feedback on the program.

\section{Statistical Analyses}

Intention-to-treat (ITT), per-protocol (PP), and frequent user analyses were conducted using IBM Statistics 25 software. The ITT sample comprised all participants who participated in the baseline assessment, whereas the PP sample comprised those who completed both the baseline and the post assessments and used the intervention at least once during the intervention period. Those who used MOOD at least once a week were considered frequent users. According to the Consolidated Standards of Reporting Trials guidelines for reporting RCTs, both ITT and PP analyses should be reported [56]. Although reporting ITT analyses might be considered as the standard analysis for clinical trials, the PP analyses provide an estimate of the true efficacy, as it only includes participants who completed the study and showed (partial) adherence. Missing values in the ITT analysis were imputed using an expectation-maximizing algorithm with 200 imputations. For each measure, we conducted ANCOVA for all samples (ITT, PP, and frequent user) with pre-post differences as the within-group factor, condition as the between-group factor, and baseline scores as the covariate to account for regression toward the mean [57]. In addition, an exploratory moderation analysis was conducted for the PP sample to identify possible moderators that affected differential symptom improvement for the 2 conditions (outcome measure: BDI-II difference scores) using the SPSS macro PROCESS by Hayes [58].

\section{Results}

\section{Baseline Characteristics}

Table 2 shows the demographic and psychopathological data of the sample at baseline. The first participant was included on May 8, 2018, and the last post assessment took place on July 
22,2018 . In total, 125 participants were included in the analyses. Of these, 63 were randomized to the CAU condition and 62 to the MOOD condition (see the study flowchart in Figure 1). Depression symptom severity was on average mild to moderate as measured by the BDI-II: 24/125 (19.2\%) had minimal symptom severity (scores 0-13), 29/125 (23.2\%) showed mild symptoms (scores 14-19), 39/125 (31.2\%) had moderate symptoms (scores 20-28), and 33/125 (26.4\%) had severe symptoms (scores 29-63). Of the sample, 49/125 (39.2\%) currently were receiving outpatient psychotherapy and 5/125 $(4.0 \%)$ were waiting for therapy.

Table 2. Demographic, psychopathological, and treatment variables along with respective statistical values $(\mathrm{N}=125)$.

\begin{tabular}{|c|c|c|}
\hline Baseline characteristics & $\operatorname{MOOD}(n=62)$ & Care-as-usual $(n=63)$ \\
\hline \multicolumn{3}{|l|}{ Demographic characteristics } \\
\hline Male, n (\%) & $15(24)$ & $18(29)$ \\
\hline Age (years), mean (SD) & $44.02(10.90)$ & $48.02(10.95)$ \\
\hline Level of school education (\% A-level), n (\%) & $37(60)$ & $36(57)$ \\
\hline \multicolumn{3}{|l|}{ Treatment variables } \\
\hline Length of distress (years), mean (SD) & $11.63(7.97)$ & $11.47(7.91)$ \\
\hline \multicolumn{3}{|l|}{ Medication, n (\%) } \\
\hline None & $38(61)$ & $35(56)$ \\
\hline Antidepressant & $20(32)$ & $22(35)$ \\
\hline Antidepressant and antipsychotics & $4(7)$ & $6(10)$ \\
\hline \multicolumn{3}{|l|}{ Treatment status, n (\%) } \\
\hline None & $27(44)$ & $28(44)$ \\
\hline In treatment & $24(39)$ & $25(40)$ \\
\hline Waiting for therapy & $2(3)$ & $3(5)$ \\
\hline Treatment expectation ${ }^{\mathrm{a}}$, mean (SD) & $5.26(1.55)$ & $5.63(1.42)$ \\
\hline \multicolumn{3}{|c|}{ Number of previous courses of psychotherapy, $n(\%)$} \\
\hline 0 & $4(7)$ & $9(14)$ \\
\hline $1-2$ & $34(55)$ & $23(37)$ \\
\hline$>2$ & $24(39)$ & $31(49)$ \\
\hline \multicolumn{3}{|l|}{ Psychometric scales, mean (SD) } \\
\hline BDI-II ${ }^{\mathrm{b}}$ (depressive symptom severity) & $22.54(11.39)$ & $22.79(11.98)$ \\
\hline PHQ-9 $9^{c}$ (depressive symptom severity) & $10.74(4.50)$ & $10.52(5.17)$ \\
\hline \multicolumn{3}{|l|}{ WHOQOL-BREF $^{\text {d }}$} \\
\hline Global & $48.39(21.88)$ & $49.80(21.47)$ \\
\hline Physical health & $55.59(18.27)$ & $57.31(19.18)$ \\
\hline Psychological & $43.55(18.74)$ & $45.90(18.48)$ \\
\hline Social relationships & $45.43(20.56)$ & $48.41(20.62)$ \\
\hline Environmental & $67.69(16.47)$ & $73.61(16.01)$ \\
\hline $\operatorname{RSE}^{\mathrm{e}}$ (self-esteem) & $26.14(6.96)$ & $26.92(6.92)$ \\
\hline
\end{tabular}

${ }^{\mathrm{a}} 1=$ not at all successful to $9=$ very successful.

b BDI-II: Beck Depression Inventory-II.

${ }^{\mathrm{c}}$ PHQ-9: Patient Health Questionnaire-9.

${ }^{\mathrm{d}}$ WHOQOL-BREF: World Health Organization Quality of Life-abbreviated version.

${ }^{\mathrm{e}} \mathrm{RSE}$ : Rosenberg Self-Esteem. 
Figure 1. Consolidated Standards of Reporting Trials flow diagram. ITT: intention-to-treat; PP: per-protocol.

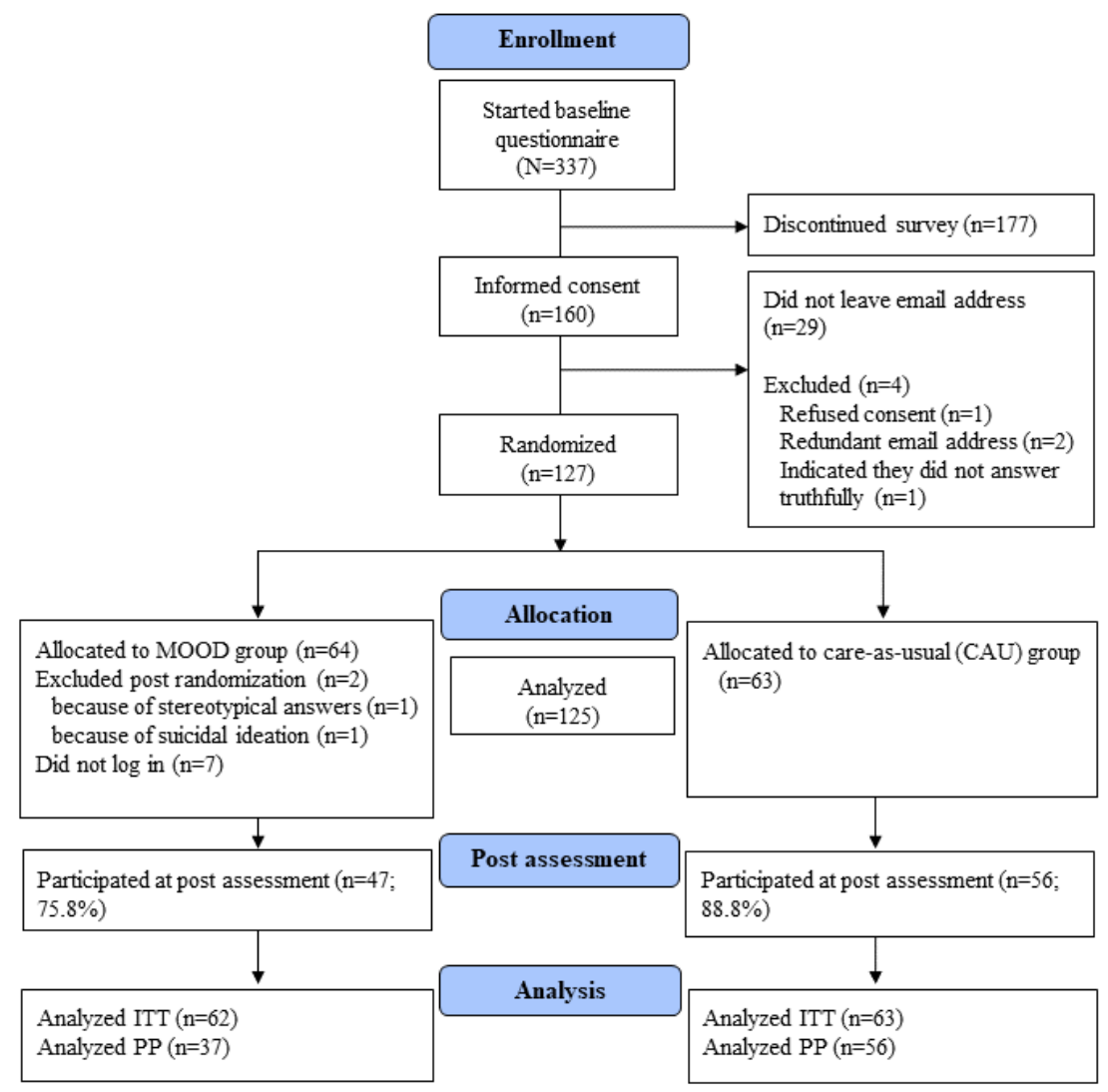

\section{Completion of Assessments and Treatment Adherence}

The completion rate of the assessments was satisfactory; $103 / 125(82.4 \%)$ completed the post assessment. There were no significant differences in completion of assessments between the intervention group and the CAU group $\left(\chi_{1,125}^{2}=3.7 ; P=.06\right)$. Completers and noncompleters were not significantly different on any baseline demographic characteristic except for 2 items regarding the presence of a diagnosis of a depressive disorder and substance or alcohol dependency. Specifically, of those who did not complete the post assessment, 6/22 (27\%) did not have a self-reported diagnosis of depression, whereas of those who completed the post assessment, $9 / 103(8.7 \%)$ did not have a self-reported diagnosis of depression $\left(\chi^{2}{ }_{1,125}=5.9 ; P=.02\right)$. Relatively more participants, $3 / 22$ (14\%), who did not complete the post assessment indicated having a diagnosis of a substance or alcohol dependency compared with those who completed the study and had such a diagnosis $\left(3 / 103,2.9 \% ; \chi^{2}{ }_{1,125}=4.6 ; P=.03\right)$.

Although the completion rate of the assessments was satisfactory, the usage of the intervention was rather low. Only
$24 / 62(39 \%)$ logged into the program at least once a week (frequent users). Of those who logged into the program, the participants completed an average of 2.90 (SD 3.13) modules. The mean time (in minutes) the users engaged with the program was 117.36 min (SD 209.05). No significant correlation could be found between duration of use and the number of completed modules with symptom improvement after the intervention $(P>.05)$.

\section{Primary Outcome}

Tables 3 and 4 shows the group differences across time of each measure for the PP sample, frequent user sample (those who logged into the program at least once a week), and ITT sample. For the primary outcome (score on the BDI-II), the results of the ANCOVA analysis did not reach statistical significance, neither for ITT nor PP or frequent user sample. Results of the paired sample $t$ tests showed a significant decline of depressive symptoms from pre to post for the intervention $\left(t_{46}=2.05 ; P=.05\right)$ and $\mathrm{CAU}\left(t_{55}=2.96 ; P=.004\right)$ groups. 
Table 3. Group differences across time; means, standard deviations, effect sizes (Cohen $d$ ) and $95 \%$ CIs of completer sample (within-group differences are denoted via superscripts).

\begin{tabular}{|c|c|c|c|c|c|c|}
\hline \multirow[t]{2}{*}{ Measurements } & \multicolumn{3}{|l|}{ MOOD } & \multicolumn{3}{|l|}{ Care-as-usual } \\
\hline & $\begin{array}{l}\text { Pre }(n=62), \\
\text { mean }(S D)\end{array}$ & $\begin{array}{l}\text { Post }(n=47) \text {, } \\
\text { mean }(S D)\end{array}$ & Cohen $d(95 \% \mathrm{CI})$ & $\begin{array}{l}\text { Pre }(n=63) \\
\text { mean }(S D)\end{array}$ & $\begin{array}{l}\text { Post }(n=56) \\
\text { mean }(S D)\end{array}$ & Cohen $d(95 \% \mathrm{CI})$ \\
\hline BDI-II ${ }^{\mathrm{a}}$ & $22.54(11.39)$ & $20.36(14.70)$ & $-0.17(-0.55 \text { to } 0.21)^{\mathrm{b}}$ & $22.79(11.99)$ & $18.68(12.79)$ & $-0.35(-0.71 \text { to } 0.02)^{\mathrm{c}}$ \\
\hline PHQ-9 ${ }^{d}$ & $10.74(4.50)$ & $10.60(6.23)$ & $-0.03(-0.41$ to 0.35$)$ & $10.52(5.17)$ & $9.11(5.64)$ & $-0.26(-0.62 \text { to } 0.01)^{\mathrm{b}}$ \\
\hline \multicolumn{7}{|l|}{ WHOQOL-BREF ${ }^{\mathrm{e}}$} \\
\hline Global & $48.39(21.88)$ & $50.00(19.85)$ & $0.08(-0.30$ to 0.46$)$ & $49.80(21.47)$ & $55.13(22.71)$ & $0.24(-0.12 \text { to } 0.60)^{\mathrm{b}}$ \\
\hline Physical health & $55.59(18.28)$ & $57.22(20.33)$ & $-0.09(-0.29$ to 0.46$)$ & $57.31(19.18)$ & $60.33(19.89)$ & $0.16(-0.21$ to 0.52$)$ \\
\hline Psychological & $43.55(18.74)$ & $46.72(20.43)$ & $-0.16(-0.22 \text { to } 0.54)^{\mathrm{f}}$ & $45.90(18.48)$ & $49.70(19.54)$ & $0.20(-0.16 \text { to } 0.56)^{\mathrm{b}}$ \\
\hline Social relationships & $45.43(20.56)$ & $48.40(23.03)$ & $-0.14(-0.24$ to 0.52$)$ & $48.41(20.62)$ & $50.30(20.59)$ & $0.09(-0.27$ to 0.45$)$ \\
\hline Environment & $67.69(16.47)$ & $70.01(14.09)$ & $-0.15(-0.23 \text { to } 0.53)^{\mathrm{b}}$ & $73.61(16.01)$ & $76.28(15.17)$ & $0.17(-0.19 \text { to } 0.53)^{\mathrm{b}}$ \\
\hline $\mathrm{RSE}^{\mathrm{g}}$ & $26.15(6.96)$ & $27.34(7.67)$ & $-0.16(-0.22 \text { to } 0.54)^{\mathrm{c}}$ & $26.92(6.12)$ & $28.68(6.74)$ & $0.27(-0.09 \text { to } 0.64)^{\mathrm{c}}$ \\
\hline
\end{tabular}

${ }^{\mathrm{a}}$ BDI-II: Beck Depression Inventory-II.

${ }^{\mathrm{b}} P \leq .05$.

${ }^{\mathrm{c}} P \leq .005$.

${ }^{\mathrm{d}}$ PHQ-9: Patient Health Questionnaire-9.

${ }^{\mathrm{e}}$ WHOQOL-BREF: World Health Organization Quality of Life-abbreviated version.

${ }^{\mathrm{f}} P \leq .01$

${ }^{g}$ RSE: Rosenberg Self-Esteem.

Table 4. Analysis of covariances with respective baseline values as covariates.

\begin{tabular}{|c|c|c|c|c|c|c|c|c|c|}
\hline \multirow[t]{2}{*}{ Measurements } & \multicolumn{2}{|c|}{ Per protocol sample $(n=93)$} & \multirow[t]{2}{*}{$P$ value } & \multicolumn{2}{|c|}{ Frequent user sample $(\mathrm{n}=80)$} & \multirow[t]{2}{*}{$P$ value } & \multicolumn{2}{|c|}{ Intention to treat $(\mathrm{n}=125)$} & \multirow[t]{2}{*}{$P$ value } \\
\hline & $F_{1,90}$ & $\eta_{\mathrm{p}}^{2}$ & & $F_{1,77}$ & $\eta_{\mathrm{p}}^{2}$ & & $F_{1,122}$ & $\eta_{\mathrm{p}}^{2}$ & \\
\hline BDI-II ${ }^{\mathrm{a}}$ & 0.24 & 0.003 & .63 & 0.24 & 0.003 & .63 & 0.94 & 0.008 & .34 \\
\hline PHQ-9 ${ }^{b}$ & 0.03 & 0.000 & .86 & 0.00 & 0.000 & .97 & 2.41 & 0.019 & .12 \\
\hline \multicolumn{10}{|l|}{ WHOQOL-BREF $^{c}$} \\
\hline Global & 0.10 & 0.001 & .75 & 0.03 & 0.000 & .87 & 1.72 & 0.014 & .19 \\
\hline Physical health & 0.66 & 0.007 & .42 & 0.04 & 0.001 & .84 & 0.37 & 0.003 & .55 \\
\hline Psychological & 0.57 & 0.006 & .45 & 1.75 & 0.022 & .19 & 0.11 & 0.001 & .75 \\
\hline Social relationships & 0.69 & 0.008 & .41 & 0.33 & 0.004 & .57 & 0.10 & 0.001 & .75 \\
\hline Environment & 0.01 & 0.000 & .95 & 0.27 & 0.003 & .61 & 0.92 & 0.008 & .34 \\
\hline $\operatorname{RSE}^{\mathrm{d}}$ & 0.879 & 0.010 & .35 & 0.51 & 0.007 & .48 & 0.44 & 0.004 & .51 \\
\hline
\end{tabular}

${ }^{\text {a} B D I-I I: ~ B e c k ~ D e p r e s s i o n ~ I n v e n t o r y-I I . ~}$

${ }^{\mathrm{b}} \mathrm{PHQ}-9$ : Patient Health Questionnaire-9.

${ }^{c}$ WHOQOL-BREF: World Health Organization Quality of Life-abbreviated version.

${ }^{\mathrm{d}}$ RSE: Rosenberg Self-Esteem.

\section{Secondary Outcomes}

Results of paired sample $t$ tests indicate a significant increase in scores on the self-esteem scale (RSE) for the intervention $\left(t_{46}=2.97 ; P=.005\right)$ and CAU $\left(t_{55}=3.04 ; P=.004\right)$ groups. There was no significant improvement across time in scores on the RSE as analyzed using an ANCOVA with baseline score as covariate (see Table 4). This is applicable to all samples analyzed (ITT, PP, and frequent user). Quality of life was assessed with the WHOQOL-BREF. Although there was a significant increase in the global score of the CAU control group $\left(t_{55}=2.46 ; P=.02\right)$, there was no significant increase in the intervention group $\left(t_{46}=1.09 ; P=.28\right)$. Paired sample $t$ tests for the subscales of the WHOQOL-BREF (physical health, 
psychological, social relationships, and environment) found a significant increase on the WHOQOL-BREF psychological (MOOD: $t_{46}=-2.86 ; P=.006$ and CAU: $t_{55}=-2.50 ; P=.02$ ) and environmental (MOOD: $t_{46}=-2.08 ; P=.04$ and CAU: $t_{55}=-2.27$; $P=.03$ ) scales for both groups. The ANCOVA analysis did not find a significant difference across groups for the WHOQOL-BREF and its subscales.

\section{Moderation Analysis}

The results for the interaction effect of the exploratory moderation analyses are summarized in Table 5. Subsequent analyses showed that participants in the intervention group who had less experience with psychotherapy showed a greater improved outcome on depression (BDI-II) compared with the CAU group $(P=.03)$. In addition, moderation analyses showed results that bordered on significance $(P=.05)$, suggesting that individuals in the intervention group who were currently receiving no other treatment had a better outcome compared with the CAU group (see Figure 2 and Table 5). Results indicate that willingness to change did not moderate treatment outcome $(P>.05)$.

Table 5. Moderators for improvement in depression (Beck Depression Inventory-II difference score, means are centered).

\begin{tabular}{llllll}
\hline Outcome parameter & Beta & SE & $t$ & $P$ value & $95 \%$ CI \\
\hline Number of past courses of psychotherapy & -.478 & 0.219 & -2.178 & .03 & -0.913 to -0.042 \\
Current treatment $^{\mathrm{a}}$ & 7.340 & 3.711 & 1.978 & .05 & -0.035 to 14.714 \\
\hline
\end{tabular}

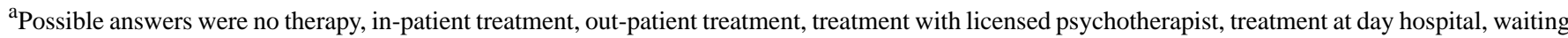
for therapy to begin, and planning on starting therapy.

Figure 2. Interaction effects of current treatment (left), number of prior treatment (right) and group allocation. The graph on the left presents the effects of current treatment $(1=y e s, 2=$ no) on symptom reduction. The graph on the right depicts how experience with psychotherapy (number of prior treatments) is related to depressive symptomatology (outcome: reduction on BDI-II). BDI-II: Beck Depression Inventory-II.

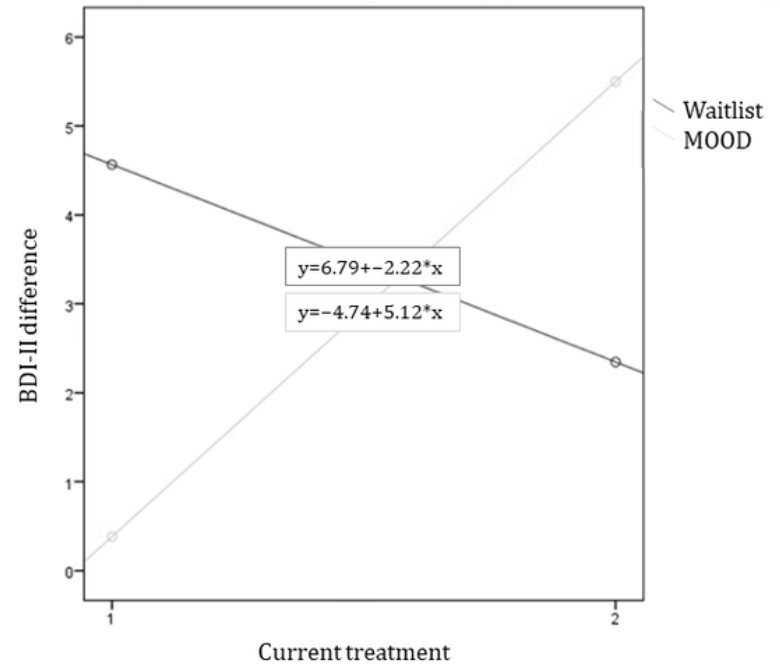

\section{Subjective Appraisal}

In Tables 6 and 7, subjective appraisals of MOOD are displayed. Overall, MOOD was positively evaluated. Of those who used the intervention, $78 \%(29 / 37)$ rated MOOD as suitable for self-help, 73\% (27/37) considered it an applicable supplement to psychotherapy, and $54 \%$ (20/37) rated the program as helpful. Furthermore, $81 \%(30 / 37)$ rated the quality of the program as excellent to good, $76 \%$ (28/37) would recommend it to a friend

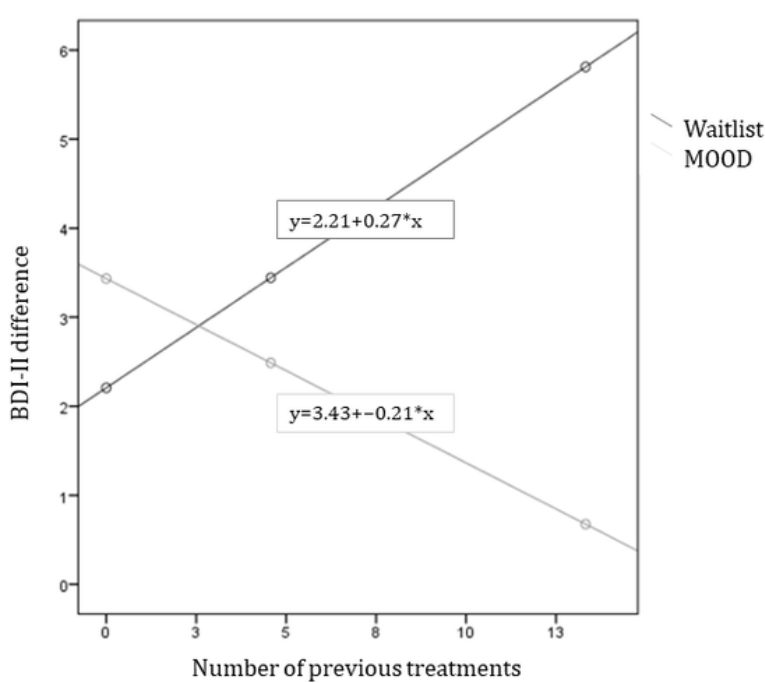

with similar problems, and $68 \%$ (25/37) would use the program again. However, only $22 \%(8 / 37)$ indicated that they were able to use MOOD regularly, $46 \%$ (17/37) had to force themselves to use the program, and only $30 \%$ (11/37) stated that their depressive symptoms decreased through using MOOD. In addition, $60 \%(18 / 30)$ rated MOOD to be equally good or better than the internet-based intervention they had previously used, and $17 \%(5 / 30)$ considered MOOD inferior. 
Table 6. Subjective appraisal of MOOD (scores: $1=$ not at all, $2=a$ little, $3=a$ lot, and $4=$ absolutely).

\begin{tabular}{|c|c|c|}
\hline Item & $\begin{array}{l}\text { MOOD condition }(n=37), \text { mean } \\
\text { (SD) }\end{array}$ & $\begin{array}{l}\text { Positive (a lot and absolutely) } \\
\text { appraisal, n (\%) }\end{array}$ \\
\hline I think the MOOD program is good for self-help and self-guidance. & $2.95(0.85)$ & $29(78)$ \\
\hline I think the contents of the program were understandable. & $1.97(0.93)$ & $35(95)$ \\
\hline I think the program was helpful. & $2.54(1.02)$ & $20(54)$ \\
\hline I was able to use the program on a regular basis during the past 6 weeks. & $1.86(0.95)$ & $8(22)$ \\
\hline I had to force myself to use the program. & $2.46(1.17)$ & $17(46)$ \\
\hline My depressive symptoms decreased because of the use of the program. & $1.97(0.93)$ & $11(30)$ \\
\hline I consider the program to be applicable as a supplement to psychotherapy. & $2.97(0.87)$ & $27(73)$ \\
\hline The program is not applicable to my depressive symptoms. & $1.76(1.07)$ & $9(24)$ \\
\hline
\end{tabular}

Table 7. Subjective appraisal of MOOD (adapted from a German questionnaire on patient satisfaction, ZUF-8 [59]).

\begin{tabular}{|c|c|c|}
\hline Item & MOOD condition $(n=37)$, mean $(S D)$ & Positive appraisal, n (\%) \\
\hline How do you rate the quality of the program? (excellent, good vs okay, not good) & $2.30(1.05)$ & $30(81)$ \\
\hline $\begin{array}{l}\text { Did you receive the type of treatment you expected to receive? (absolutely, a } \\
\text { lot vs a little, not at all) }\end{array}$ & $3.16(0.90)$ & $25(68)$ \\
\hline $\begin{array}{l}\text { To what extent did the program help you cope with your problems? (absolutely, } \\
\text { a lot vs a little, not at all) }\end{array}$ & $2.76(1.14)$ & $19(51)$ \\
\hline $\begin{array}{l}\text { Would you recommend the program to a friend with similar symptoms? (yes, } \\
\text { probably yes vs probably not, no) }\end{array}$ & $3.32(0.97)$ & $28(76)$ \\
\hline $\begin{array}{l}\text { How happy are you about the extent of the help you have received through using } \\
\text { the program? (very satisfied, mostly satisfied vs somewhat dissatisfied, dissatis- } \\
\text { fied) }\end{array}$ & $3.16(1.07)$ & $21(57)$ \\
\hline $\begin{array}{l}\text { Did the program help you cope with your problems more successfully? (abso- } \\
\text { lutely, a lot vs a little, not at all) }\end{array}$ & $2.65(1.36)$ & $21(57)$ \\
\hline $\begin{array}{l}\text { How satisfied are you with the program in general? (very satisfied, mostly satis- } \\
\text { fied vs somewhat unsatisfied, unsatisfied) }\end{array}$ & $2.57(1.28)$ & $25(68)$ \\
\hline Would you use the program again? (Yes, probably yes vs probably not, no) & $3.35(1.11)$ & $25(68)$ \\
\hline
\end{tabular}

\section{Discussion}

\section{Principal Findings}

The treatment gap in the treatment of depressive disorders is a significant issue [8] and might be narrowed by providing effective internet-based interventions. Self-guided internet-based interventions can be especially useful because therapists are a scarce resource. Furthermore, depressive episodes tend to reoccur [60], but treatment barriers are stable, which means that affected individuals are repeatedly confronted with the problem of the undersupply of therapists. In our study, we aimed to investigate whether the use of a new self-guided internet-based intervention called MOOD over a period of 6 weeks would lead to a significant reduction in depressive symptoms compared with a CAU group (that got access to MOOD after completion of the post assessment) in a sample of individuals who had previously and/or currently received therapy (most of the participants had also already received a different internet-based intervention).

In our analyses, we found no significant difference across groups in any outcome variable (for ITT, PP, and frequent users). Contrary to our hypothesis, the group that received MOOD over

the intervention period did not significantly improve in depressive symptoms compared with the CAU group. Both groups significantly improved over time and, interestingly, the difference here was more pronounced for the CAU group for the primary outcome, which was the BDI-II. Both groups significantly improved over time in levels of self-esteem and quality of life, but this effect again was not different across groups. These findings are different from previous trials investigating the effectiveness of self-guided internet-based interventions. Here, it was found that self-guided internet-based interventions were effective in treating depressive symptoms at a small to medium effect size $[21,23]$. Certainly, it is possible that this difference between ours and previous results might stem from a publication bias. Furthermore, self-guided internet-based interventions were found to be more effective in individuals currently not seeing a psychotherapist $[33,61]$. Results of this study support this finding with a $P$ value of .05 , which indicates that individuals in the intervention group who benefited more from the program were currently not receiving other psychotherapy. However, this result was not statistically significant. The number of participants who were in treatment while using MOOD amounted to $39 \%$, and these individuals had a smaller symptom reduction compared with those who were not in treatment. This result indicates that internet-based 
interventions appear to be more useful to individuals who are currently not receiving psychotherapy. More than $70 \%$ of participants in the intervention group considered MOOD to be a suitable supplement to psychotherapy. However, this is more likely to be the case if the combination of internet-based interventions and psychotherapy is addressed in therapy, and the integration is actively promoted, as in blended therapy $[62,63,64]$.

Another interesting finding was that the participants' past experience with psychotherapy had a significant effect on treatment outcome. Here, results indicated that individuals with less experience benefited more than those with more experience. Our sample mainly comprised individuals with a relatively long history of depression; most of them had undergone multiple courses of psychotherapy in the past. Only $10.4 \%$ of the overall sample had not previously received psychotherapy. Also, $82.9 \%$ had previously used a comparable self-guided internet-based intervention within the framework of another trial [33]. Our results support the assumption that individuals who have already used a very similar internet-based intervention and have a high level of psychotherapeutic experience may not benefit from another self-guided Web-based intervention. Perhaps they should receive personalized support, such as a guided internet-based intervention. It is conceivable that individuals who have suffered from depression for a long time and who already have had significant treatment experience will not learn much that is unknown or new to them in a subsequent intervention. Another explanation might be that individuals who have not benefited from conventional psychotherapy and, therefore, have continued to look for other forms of treatment also will not benefit from internet-based treatment. The fact that people with a long history of depression usually suffer from residual symptoms that are difficult to treat is a well-known prognostic factor and therefore is presumably another reason for the nonsignificant group differences we found in our sample $[65,66]$. Another important point is that patient's expectations of the outcome of therapy is considered a key determinant of the actual therapy outcome $[67,68]$. Many patients with a long history of a mental disorder and extensive therapy experience are likely to have lower expectations and hopes regarding treatment outcome. This naturally applies to other forms of therapy too and is not confined to internet-based interventions. However, it could be a further reason for the lack of treatment effects found in our study. Despite this, based on the results presented above, we come to the conclusion that unguided internet-based interventions should mainly serve as a first step in treatment and are not suitable for individuals with recurrent or residual symptoms. Our findings, therefore, support stepped care approaches, in which self-guided internet-based interventions are used in early phases of illness and in patients without a chronification of symptoms [69-71]. The key idea behind these stepped models is that patients should first be treated at the lowest level (to avoid psychiatrization and self-stigma) and only proceed to more advanced care when symptoms are severe or do not improve with (guided) self-help. It has been found that stepped care approaches can be effective and resource-saving in the treatment of common mental disorders, such as depression [72]. Another potential implication is that programs for individuals with therapeutic experience have to be more elaborated to be effective. For instance, they could consider motives of participants [48] or be personalized in other ways.

\section{Strengths and Limitations}

To participate in the study, neither a (verified) diagnosis of depression nor a minimum severity of depression symptoms was necessary, which resulted in heterogeneity of depression levels. Therefore, on the one hand, a wider range of individuals with desire for treatment for depression was reached, regardless of whether they met the criteria of a diagnosis or not. On the other hand, it has been found that samples of severely depressed participants benefit more from low-intensity psychological interventions than samples of mildly depressed participants [32], which might be because those with severe depression have more room to improve. This finding, however, contrasts with results of Karyotaki et al [21] who found that self-guided internet-based interventions are effective regardless of symptom severity. Interestingly, within another study by Karyotaki et al [73], it was found that individuals with more severe baseline symptoms were more likely to improve than individuals with less severe baseline symptoms after treatment with guided internet-based interventions, which means that the findings are still ambiguous in this respect. Apart from this, broad inclusion criteria may result in a type I error and, thereby, lead to an underestimation of the intervention's treatment potential. Another limitation might be the sample size in our study. Our power analysis was based on a medium effect, and we did not consider expected dropout in our sample size calculation, which could have led to an overestimation, as Karyotaki et al [21] only found a small effect $(\mathrm{g}=0.27)$ for self-guided interventions. To detect such a small effect, a larger sample would have been necessary.

A strength of the study is the high completion rate of the assessments-only $17.6 \%$ did not participate or complete the post assessment. Despite the high completion rate, the treatment adherence was rather low; only $39 \%$ were able to use the program on a regular basis (at least once a week), and the average time of program usage was 117.36 min. Contrary to the results of other studies, there was no significant correlation between duration of use and therapy outcome [21,74]. Interestingly, the percentage of participants who self-reported a regular use was minor (22\%) compared with the percentage of the frequent users as defined by us. It is conceivable that depressive users themselves define much higher standards for regular use. If they do not meet these demands, this leads to disappointment and negative feelings. This point can be discussed in the context of depression-specific negative cognitive distortions, namely, (dysfunctional) perfectionism and all-or-nothing thinking [75,76]. Individuals with depression, who have these thought distortions, often think that an expectation task is either fulfilled perfectly (100\%) or not at all. In our case, this could mean that the participants rated themselves or their use of the program worse than they actually did because of their depressive way of thinking. Strategies that improve adherence in self-guided internet-based interventions (such as sending reminders) have been found to have a positive effect on treatment outcome [77]. However, in our study, we did send frequent reminders to participants to use the intervention. Finally, as the study had limited financial 
resources, follow-up assessments were not possible. For this reason, no conclusion can be drawn about long-term effects.

\section{Conclusions}

The efficacy of unguided internet-based interventions has been proven in various trials. However, it is unclear whether internet-based interventions are beneficial for individuals with a long history of depression and greater experience with psychotherapy and for those who have already undergone a different unguided Web-based intervention. With this sample, we could not verify the efficacy of the new self-help internet-based intervention MOOD; however, 2 interesting findings with implications for the future were identified. Greater experience with psychotherapy and (at trend level) current treatment reduced the effects of the intervention on depressive symptoms. We conclude that unguided internet-based interventions might be appropriate as a first step in treatment but not for individuals who already have much experience with psychotherapy or internet-based interventions.

\section{Acknowledgments}

The study was partly supported by a grant of the Swiss National Science Foundation to SW and SM (Grant Number 159384) and a fellowship of the Swiss National Science Foundation to SW (Number 177678). The study was not externally funded. It was financially supported by private donations to the working group and by overhead funds of the institution.

\section{Conflicts of Interest}

None declared.

\section{Multimedia Appendix 1}

CONSORT - EHEALTH checklist (V 1.6.1).

[PDF File (Adobe PDF File)107 KB-Multimedia Appendix 1]

\section{References}

1. World Health Organization. 2017. Depression and Other Common Mental Disorders Global Health Estimates URL: $\underline{\text { https:/ }}$ lapps.who.int/iris/bitstream/handle/10665/254610/WHO-MSD-MER-2017.2-eng.pdf [accessed 2019-08-15]

2. Lim GY, Tam WW, Lu Y, Ho CS, Zhang MW, Ho RC. Prevalence of depression in the community from 30 countries between 1994 and 2014. Sci Rep 2018 Feb 12;8(1):2861 [FREE Full text] [doi: 10.1038/s41598-018-21243-x] [Medline: 29434331]

3. Sobocki P, Jönsson B, Angst J, Rehnberg C. Cost of depression in Europe. J Ment Health Policy Econ 2006 Jun;9(2):87-98. [Medline: 17007486 ]

4. Lépine JP, Briley M. The increasing burden of depression. Neuropsychiatr Dis Treat 2011;7(Suppl 1):3-7 [FREE Full text] [doi: 10.2147/NDT.S19617] [Medline: 21750622]

5. Cuijpers P, Berking M, Andersson G, Quigley L, Kleiboer A, Dobson KS. A meta-analysis of cognitive-behavioural therapy for adult depression, alone and in comparison with other treatments. Can J Psychiatry 2013 Jul;58(7):376-385. [doi: 10.1177/070674371305800702] [Medline: 23870719]

6. Cuijpers P, Andersson G, Donker T, van Straten A. Psychological treatment of depression: results of a series of meta-analyses. Nord J Psychiatry 2011 Dec;65(6):354-364. [doi: 10.3109/08039488.2011.596570] [Medline: 21770842]

7. Cuijpers P, Sijbrandij M, Koole SL, Andersson G, Beekman AT, Reynolds CF. The efficacy of psychotherapy and pharmacotherapy in treating depressive and anxiety disorders: a meta-analysis of direct comparisons. World Psychiatry 2013 Jun;12(2):137-148 [FREE Full text] [doi: 10.1002/wps.20038] [Medline: 23737423]

8. Kohn R, Saxena S, Levav I, Saraceno B. The treatment gap in mental health care. Bull World Health Organ 2004 Nov;82(11):858-866 [FREE Full text] [doi: 10.1590/S0042-96862004001100011] [Medline: 15640922]

9. Andrade LH, Alonso J, Mneimneh Z, Wells JE, Al-Hamzawi A, Borges G, et al. Barriers to mental health treatment: results from the WHO World Mental Health surveys. Psychol Med 2014 Apr;44(6):1303-1317 [FREE Full text] [doi: 10.1017/S0033291713001943] [Medline: 23931656]

10. Carey M, Jones K, Meadows G, Sanson-Fisher R, D'Este C, Inder K, et al. Accuracy of general practitioner unassisted detection of depression. Aust N Z J Psychiatry 2014 Jun;48(6):571-578 [FREE Full text] [doi: 10.1177/0004867413520047] [Medline: 24413807]

11. Clement S, Schauman O, Graham T, Maggioni F, Evans-Lacko S, Bezborodovs N, et al. What is the impact of mental health-related stigma on help-seeking? A systematic review of quantitative and qualitative studies. Psychol Med 2015 Jan;45(1):11-27. [doi: 10.1017/S0033291714000129] [Medline: 24569086]

12. Gulliver A, Griffiths KM, Christensen H. Perceived barriers and facilitators to mental health help-seeking in young people: a systematic review. BMC Psychiatry 2010 Dec 30;10:113 [FREE Full text] [doi: 10.1186/1471-244X-10-113] [Medline: 21192795] 
13. Mohr DC, Ho J, Duffecy J, Baron KG, Lehman KA, Jin L, et al. Perceived barriers to psychological treatments and their relationship to depression. J Clin Psychol 2010 Apr;66(4):394-409 [FREE Full text] [doi: 10.1002/jclp.20659] [Medline: 20127795]

14. Lal S, Adair CE. E-mental health: a rapid review of the literature. Psychiatr Serv 2014 Jan 1;65(1):24-32. [doi: 10.1176/appi.ps.201300009] [Medline: 24081188]

15. Richards D, Viganó N. Online counseling: a narrative and critical review of the literature. J Clin Psychol 2013 Sep;69(9):994-1011. [doi: 10.1002/jclp.21974] [Medline: 23630010]

16. Andersson G, Titov N. Advantages and limitations of internet-based interventions for common mental disorders. World Psychiatry 2014 Feb;13(1):4-11 [FREE Full text] [doi: 10.1002/wps.20083] [Medline: 24497236]

17. Hedman E, Ljótsson B, Lindefors N. Cognitive behavior therapy via the internet: a systematic review of applications, clinical efficacy and cost-effectiveness. Expert Rev Pharmacoecon Outcomes Res 2012 Dec;12(6):745-764. [doi: 10.1586/erp.12.67] [Medline: 23252357]

18. Carlbring P, Andersson G, Cuijpers P, Riper H, Hedman-Lagerlöf E. Internet-based vs face-to-face cognitive behavior therapy for psychiatric and somatic disorders: an updated systematic review and meta-analysis. Cogn Behav Ther 2018 Jan;47(1):1-18. [doi: 10.1080/16506073.2017.1401115] [Medline: 29215315]

19. Ebert DD, Donkin L, Andersson G, Andrews G, Berger T, Carlbring P, et al. Does internet-based guided-self-help for depression cause harm? An individual participant data meta-analysis on deterioration rates and its moderators in randomized controlled trials. Psychol Med 2016 Oct;46(13):2679-2693 [FREE Full text] [doi: 10.1017/S0033291716001562] [Medline: 27649340]

20. Karyotaki E, Ebert DD, Donkin L, Riper H, Twisk J, Burger S, et al. Do guided internet-based interventions result in clinically relevant changes for patients with depression? An individual participant data meta-analysis. Clin Psychol Rev 2018 Jul;63:80-92. [doi: 10.1016/j.cpr.2018.06.007] [Medline: 29940401]

21. Karyotaki E, Riper H, Twisk J, Hoogendoorn A, Kleiboer A, Mira A, et al. Efficacy of self-guided internet-based cognitive behavioral therapy in the treatment of depressive symptoms: a meta-analysis of individual participant data. JAMA Psychiatry 2017 Apr 1;74(4):351-359. [doi: 10.1001/jamapsychiatry.2017.0044] [Medline: 28241179]

22. Lüdtke T, Westermann S, Pult LK, Schneider BC, Pfuhl G, Moritz S. Evaluation of a brief unguided psychological online intervention for depression: a controlled trial including exploratory moderator analyses. Internet Interv 2018 Sep;13:73-81 [FREE Full text] [doi: 10.1016/j.invent.2018.06.004] [Medline: 30206522]

23. Richards D, Richardson T. Computer-based psychological treatments for depression: a systematic review and meta-analysis. Clin Psychol Rev 2012 Jun;32(4):329-342. [doi: 10.1016/j.cpr.2012.02.004] [Medline: 22466510]

24. Schröder J, Berger T, Westermann S, Klein JP, Moritz S. Internet interventions for depression: new developments. Dialogues Clin Neurosci 2016 Jun;18(2):203-212 [FREE Full text] [Medline: 27489460]

25. Stolz T, Schulz A, Krieger T, Vincent A, Urech A, Moser C, et al. A mobile app for social anxiety disorder: a three-arm randomized controlled trial comparing mobile and PC-based guided self-help interventions. J Consult Clin Psychol 2018 Jun;86(6):493-504. [doi: 10.1037/ccp0000301] [Medline: 29781648]

26. Andersson G, Cuijpers P, Carlbring P, Riper H, Hedman E. Guided internet-based vs face-to-face cognitive behavior therapy for psychiatric and somatic disorders: a systematic review and meta-analysis. World Psychiatry 2014 Oct;13(3):288-295 [FREE Full text] [doi: 10.1002/wps.20151] [Medline: 25273302]

27. van Ballegooijen W, Cuijpers P, van Straten A, Karyotaki E, Andersson G, Smit JH, et al. Adherence to internet-based and face-to-face cognitive behavioural therapy for depression: a meta-analysis. PLoS One 2014;9(7):e100674 [FREE Full text] [doi: 10.1371/journal.pone.0100674] [Medline: 25029507]

28. Melville KM, Casey LM, Kavanagh DJ. Dropout from internet-based treatment for psychological disorders. Br J Clin Psychol 2010 Nov;49(Pt 4):455-471. [doi: 10.1348/014466509X472138] [Medline: 19799804]

29. Karyotaki E, Kleiboer A, Smit F, Turner DT, Pastor AM, Andersson G, et al. Predictors of treatment dropout in self-guided web-based interventions for depression: an 'individual patient data' meta-analysis. Psychol Med 2015 Oct;45(13):2717-2726. [doi: 10.1017/S0033291715000665] [Medline: 25881626]

30. Berger T, Hämmerli K, Gubser N, Andersson G, Caspar F. Internet-based treatment of depression: a randomized controlled trial comparing guided with unguided self-help. Cogn Behav Ther 2011;40(4):251-266. [doi: 10.1080/16506073.2011.616531] [Medline: 22060248]

31. Baumeister H, Reichler L, Munzinger M, Lin J. The impact of guidance on internet-based mental health interventions a systematic review. Internet Interv 2014 Oct;1(4):205-215. [doi: 10.1016/j.invent.2014.08.003]

32. Bower P, Kontopantelis E, Sutton A, Kendrick T, Richards DA, Gilbody S, et al. Influence of initial severity of depression on effectiveness of low intensity interventions: meta-analysis of individual patient data. Br Med J 2013 Feb 26;346:f540 [FREE Full text] [doi: 10.1136/bmj.f540] [Medline: 23444423]

33. Klein JP, Berger T, Schröder J, Späth C, Meyer B, Caspar F, et al. Effects of a psychological internet intervention in the treatment of mild to moderate depressive symptoms: results of the EVIDENT study, a randomized controlled trial. Psychother Psychosom 2016;85(4):218-228. [doi: 10.1159/000445355] [Medline: 27230863] 
34. Dozois DJ, Westra HA, Collins KA, Fung TS, Garry JK. Stages of change in anxiety: psychometric properties of the University of Rhode Island change assessment (URICA) scale. Behav Res Ther 2004 Jun;42(6):711-729. [doi: 10.1016/S0005-7967(03)00193-1] [Medline: 15081886]

35. Faul F, Erdfelder E, Lang A, Buchner AG. G*Power 3: a flexible statistical power analysis program for the social, behavioral, and biomedical sciences. Behav Res Methods 2007 May;39(2):175-191. [doi: 10.3758/BF03193146] [Medline: 17695343]

36. Urbaniak G, Plous S. Research Randomizer. 2018. URL: https://www.randomizer.org/ [accessed 2018-04-23]

37. Hollon SD, Thase ME, Markowitz JC. Treatment and prevention of depression. Psychol Sci Public Interest 2002 Nov;3(2):39-77. [doi: 10.1111/1529-1006.00008] [Medline: 26151569]

38. Cuijpers P, van Straten A, Warmerdam L. Behavioral activation treatments of depression: a meta-analysis. Clin Psychol Rev 2007 Apr;27(3):318-326. [doi: 10.1016/j.cpr.2006.11.001] [Medline: 17184887]

39. Hofmann SG, Sawyer AT, Witt AA, Oh D. The effect of mindfulness-based therapy on anxiety and depression: a meta-analytic review. J Consult Clin Psychol 2010 Apr;78(2):169-183 [FREE Full text] [doi: 10.1037/a0018555] [Medline: 20350028]

40. Khoury B, Lecomte T, Fortin G, Masse M, Therien P, Bouchard V, et al. Mindfulness-based therapy: a comprehensive meta-analysis. Clin Psychol Rev 2013 Aug;33(6):763-771. [doi: 10.1016/j.cpr.2013.05.005] [Medline: 23796855]

41. Teasdale JD, Segal ZV, Williams JM, Ridgeway VA, Soulsby JM, Lau MA. Prevention of relapse/recurrence in major depression by mindfulness-based cognitive therapy. J Consult Clin Psychol 2000 Aug;68(4):615-623. [doi: 10.1037/0022-006X.68.4.615] [Medline: 10965637]

42. Segrin C. Social skills deficits associated with depression. Clin Psychol Rev 2000 Apr;20(3):379-403. [doi: 10.1016/S0272-7358(98)00104-4] [Medline: 10779900]

43. Franzen PL, Buysse DJ. Sleep disturbances and depression: risk relationships for subsequent depression and therapeutic implications. Dialogues Clin Neurosci 2008;10(4):473-481 [FREE Full text] [Medline: 19170404]

44. Murphy MJ, Peterson MJ. Sleep disturbances in depression. Sleep Med Clin 2015 Mar;10(1):17-23 [FREE Full text] [doi: 10.1016/j.jsmc.2014.11.009] [Medline: 26055669]

45. Ellis A. Reason And Emotion In Psychotherapy. New York, United States: Citadel; 1994.

46. Rüdiger H, Pfingsten U. Gruppentraining Sozialer Kompetenzen GSK. Weinheim, Germany: Beltz Verlag; 2015.

47. Kaluza G. Stressbewältigung: Trainingsmanual zur Psychologischen Gesundheitsförderung. Berlin, Heidelberg: Springer-Verlag; 2015.

48. Westermann S, Moritz S, Berger T. Internet- und mobilbasierte interventionen bei schizophrenie. Verhaltenstherapie 2017 Aug 18;27(3):181-189. [doi: 10.1159/000479335]

49. Beck AT, Steer RA, Brown GK. Manual for the Beck Depression Inventory-II. San Antonio, TX: Psychological Corporation; 1996.

50. Smarr KL, Keefer AL. Measures of depression and depressive symptoms: Beck Depression Inventory-II (BDI-II), Center for Epidemiologic Studies Depression Scale (CES-D), Geriatric Depression Scale (GDS), Hospital Anxiety and Depression Scale (HADS), and Patient Health Questionnaire-9 (PHQ-9). Arthritis Care Res (Hoboken) 2011 Nov;63(Suppl 11):S454-S466 [FREE Full text] [doi: 10.1002/acr.20556] [Medline: 22588766]

51. Kroenke K, Spitzer RL, Williams JB. The PHQ-9: validity of a brief depression severity measure. J Gen Intern Med 2001 Sep;16(9):606-613 [FREE Full text] [doi: 10.1046/j.1525-1497.2001.016009606.x] [Medline: 11556941]

52. Rosenberg M. York University. 1965. Rosenberg Self-Esteem Scale (RSE) URL: https://www.yorku.ca/rokada/psyctest/ rosenbrg.pdf [accessed 2019-08-16]

53. Group TW. Development of the World Health Organization WHOQOL-BREF quality of life assessment. The WHOQOL group. Psychol Med 1998 May;28(3):551-558. [doi: 10.1017/S0033291798006667] [Medline: 9626712]

54. Skevington SM, Lotfy M, O'Connell KA, WHOQOL Group. The World Health Organization's WHOQOL-BREF quality of life assessment: psychometric properties and results of the international field trial. A report from the WHOQOL group. Qual Life Res 2004 Mar;13(2):299-310. [doi: 10.1023/B:QURE.0000018486.91360.00] [Medline: 15085902]

55. Schmidt J, Lamprecht F, Wittmann WW. [Satisfaction with inpatient management. Development of a questionnaire and initial validity studies]. Psychother Psychosom Med Psychol 1989 Jul;39(7):248-255. [Medline: 2762479]

56. Ranganathan P, Pramesh C, Aggarwal R. Common pitfalls in statistical analysis: absolute risk reduction, relative risk reduction, and number needed to treat. Perspect Clin Res 2016;7(1):51-53 [FREE Full text] [doi: 10.4103/2229-3485.173773] [Medline: 26952180]

57. Vickers AJ, Altman DG. Statistics notes: analysing controlled trials with baseline and follow up measurements. Br Med J 2001 Nov 10;323(7321):1123-1124 [FREE Full text] [doi: 10.1136/bmj.323.7321.1123] [Medline: 11701584]

58. Hayes AF. Introduction to Mediation, Moderation, and Conditional Process Analysis: A Regression-Based Approach. New York: Guildford Press; 2013.

59. Schmidt J, Wittmann WW. Fragebogen zur Messung der Patientenzufriedenheit. In: Diagnostische Verfahren in der Psychotherapie. Göttingen: Hogrefe; 2002:392-396.

60. Burcusa SL, Iacono WG. Risk for recurrence in depression. Clin Psychol Rev 2007 Dec;27(8):959-985 [FREE Full text] [doi: 10.1016/j.cpr.2007.02.005] [Medline: 17448579]

61. Moritz S, Schilling L, Hauschildt M, Schröder J, Treszl A. A randomized controlled trial of internet-based therapy in depression. Behav Res Ther 2012 Aug;50(7-8):513-521. [doi: 10.1016/j.brat.2012.04.006] [Medline: 22677231] 
62. Erbe D, Eichert HC, Riper H, Ebert DD. Blending face-to-face and internet-based interventions for the treatment of mental disorders in adults: systematic review. J Med Internet Res 2017 Sep 15;19(9):e306 [FREE Full text] [doi: 10.2196/jmir.6588] [Medline: 28916506]

63. Wentzel J, van der Vaart R, Bohlmeijer ET, van Gemert-Pijnen JE. Mixing online and face-to-face therapy: how to benefit from blended care in mental health care. JMIR Ment Health 2016 Feb 9;3(1):e9 [FREE Full text] [doi: 10.2196/mental.4534] [Medline: 26860537]

64. Berger T, Krieger T, Sude K, Meyer B, Maercker A. Evaluating an e-mental health program ("deprexis") as adjunctive treatment tool in psychotherapy for depression: Results of a pragmatic randomized controlled trial. J Affect Disord 2018 Feb;227:455-462. [doi: 10.1016/j.jad.2017.11.021] [Medline: 29154168]

65. Culpepper L, Muskin PR, Stahl SM. Major depressive disorder: understanding the significance of residual symptoms and balancing efficacy with tolerability. Am J Med 2015 Sep;128(9 Suppl):S1-15. [doi: 10.1016/j.amjmed.2015.07.001] [Medline: 26337210]

66. Israel JA. The impact of residual symptoms in major depression. Pharmaceuticals (Basel) 2010 Aug 3;3(8):2426-2440 [FREE Full text] [doi: 10.3390/ph3082426] [Medline: 27713362]

67. Constantino MJ, Arnkoff DB, Glass CR, Ametrano RM, Smith JZ. Expectations. J Clin Psychol 2011 Feb;67(2):184-192. [doi: 10.1002/jclp.20754] [Medline: 21128304]

68. Greenberg RP, Constantino MJ, Bruce N. Are patient expectations still relevant for psychotherapy process and outcome? Clin Psychol Rev 2006 Oct;26(6):657-678. [doi: 10.1016/j.cpr.2005.03.002] [Medline: 15908088]

69. Scogin FR, Hanson A, Welsh D. Self-administered treatment in stepped-care models of depression treatment. J Clin Psychol 2003 Mar;59(3):341-349. [doi: 10.1002/jclp.10133] [Medline: 12579549]

70. van Straten A, Seekles W, van't Veer-Tazelaar NJ, Beekman AT, Cuijpers P. Stepped care for depression in primary care: what should be offered and how? Med J Aust 2010 Jun 7;192(11 Suppl):S36-S39. [Medline: 20528706]

71. Lambert M, Karow A, Deister A, Ohm G, Bargel S, Peter H, et al. RECOVER: modell der sektorenübergreifend-koordinierten, schweregrad-gestuften, evidenzbasierten versorgung psychischer erkrankungen. In: Amelung VE, Eble S, Hildebrandt H, Knieps F, Lägel R, Ozegowski S, et al, editors. Innovationsfonds: Impulse Für Das Deutsche Gesundheitssystem. Hannover: Medizinisch Wissenschaftliche Verlagsgesellschaft; 2017:252-265.

72. Salomonsson S, Santoft F, Lindsäter E, Ejeby K, Ljótsson B, Öst LG, et al. Stepped care in primary care - guided self-help and face-to-face cognitive behavioural therapy for common mental disorders: a randomized controlled trial. Psychol Med 2018 Jul;48(10):1644-1654. [doi: 10.1017/S0033291717003129] [Medline: 29095133]

73. Karyotaki E, Kemmeren L, Riper H, Twisk J, Hoogendoorn A, Kleiboer A, et al. Is self-guided internet-based cognitive behavioural therapy (iCBT) harmful? An individual participant data meta-analysis. Psychol Med 2018 Nov;48(15):2456-2466 [FREE Full text] [doi: 10.1017/S0033291718000648] [Medline: 29540243]

74. Donkin L, Christensen H, Naismith SL, Neal B, Hickie IB, Glozier N. A systematic review of the impact of adherence on the effectiveness of e-therapies. J Med Internet Res 2011 Aug 5;13(3):e52 [FREE Full text] [doi: 10.2196/jmir.1772] [Medline: 21821503]

75. Jelinek L, Hauschildt M, Wittekind CE, Schneider BC, Kriston L, Moritz S. Efficacy of metacognitive training for depression: a randomized controlled trial. Psychother Psychosom 2016;85(4):231-234. [doi: 10.1159/000443699] [Medline: 27230865]

76. Teasdale J, Scott J, Moore R, Hayhurst H, Pope M, Paykel E. How does cognitive therapy prevent relapse in residual depression? Evidence from a controlled trial. J Consult Clin Psychol 2001 Jun;69(3):347-357. [doi: 10.1037//0022-006X.69.3.347] [Medline: 11495165]

77. Titov N, Dear BF, Johnston L, Lorian C, Zou J, Wootton B, et al. Improving adherence and clinical outcomes in self-guided internet treatment for anxiety and depression: randomised controlled trial. PLoS One 2013;8(7):e62873 [REE Full text] [doi: 10.1371/journal.pone.0062873] [Medline: 23843932]

\author{
Abbreviations \\ ABC (-protocol): activating event, beliefs, consequence \\ ANCOVA: analysis of covariances \\ BDI-II: Beck Depression Inventory-II \\ CAU: care-as-usual \\ CBT: cognitive behavioral therapy \\ ITT: intention-to-treat \\ MD: major depression \\ PHQ-9: Patient Health Questionnaire-9 \\ PP: per-protocol \\ RCT: randomized controlled trial \\ RSE: Rosenberg Self-Esteem \\ URICA: University of Rhode Island Change Assessment \\ WHO: World Health Organization
}


WHOQOL-BREF: World Health Organization Quality of Life-abbreviated version ZUF-8: Fragebogen zur Patientenzufriedenheit (patient satisfaction questionnaire)

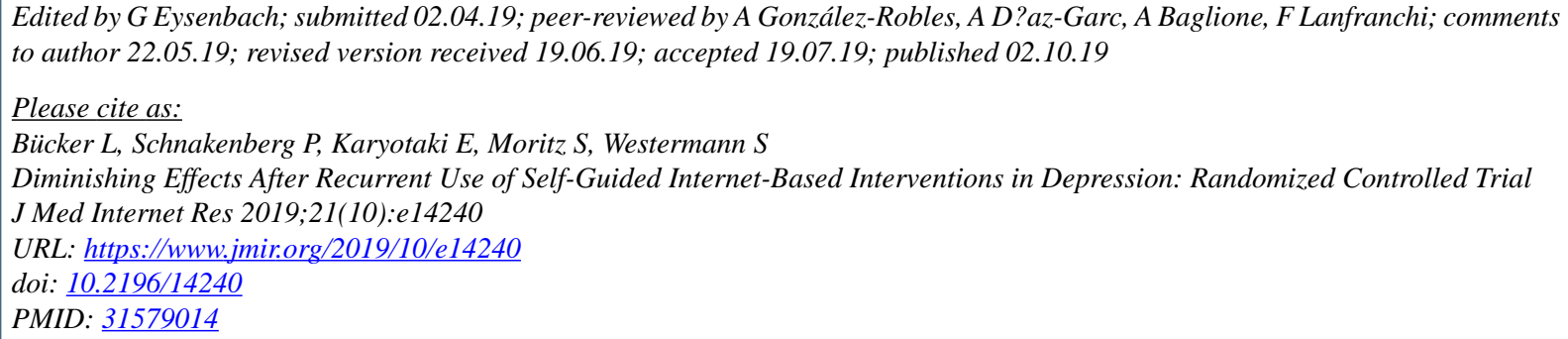

CLara Bücker, Patricia Schnakenberg, Eirini Karyotaki, Steffen Moritz, Stefan Westermann. Originally published in the Journal of Medical Internet Research (http://www.jmir.org), 02.10.2019 This is an open-access article distributed under the terms of the Creative Commons Attribution License (https://creativecommons.org/licenses/by/4.0/), which permits unrestricted use, distribution, and reproduction in any medium, provided the original work, first published in the Journal of Medical Internet Research, is properly cited. The complete bibliographic information, a link to the original publication on http://www.jmir.org/, as well as this copyright and license information must be included. 